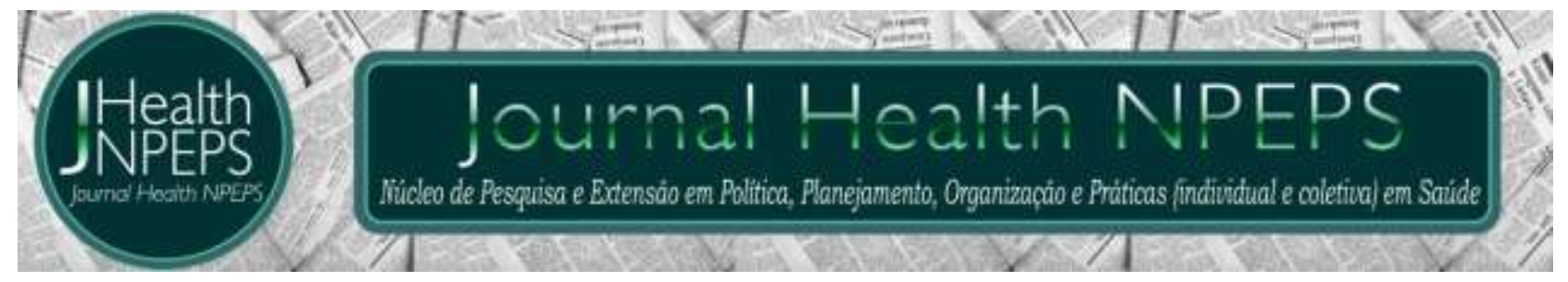

http://dx.doi.org/10.30681/252610103123

ARTIGO ORIGINAL

\title{
Relación de calidad de vida y autocuidado en pacientes adultos con cáncer
}

\author{
Relationship of quality of life and self-care in adult patients with cancer
}

\section{Relação da qualidade de vida e autocuidado em pacientes adultos com câncer}

\section{Alejandro Torres-Reyes ${ }^{1}$, Inés Tenahua-Quitl ${ }^{2}$, María Araceli García-López ${ }^{3}$, Erika Pérez-Noriega ${ }^{4}$, Clemente Cordero-Sánchez ${ }^{5}$, Nataly Ramos-Durán ${ }^{6}$, Francisco Adrian Morales-Castillo ${ }^{7}$}

\begin{abstract}
RESUMEN
Objetivo: determinar la relación de calidad de vida y capacidades de autocuidado en pacientes adultos con cáncer. Método: estudio correlacional, analítico, no probabilístico por conveniencia con una muestra constituida por 51 pacientes adultos diagnosticados con cáncer que aceptarón participar en el estudio mediante un consentimiento informado. Se aplicó una cédula de datos sociodemográficos y dos instrumentos: Calidad de Vida WHOQOL-BREF y Capacidad de Agencia de Autocuidado. Resultados: la edad promedio fue de 50.32 años, predomino el género femenino (60\%), los tipos de cáncer con mayor prevalencia fueron: cáncer de mama (20\%), de próstata (16\%) y linfoma no hodking (12\%), otros tipos (52\%). El $80 \%$ estuvo en estadio III TNM, con respecto a calidad de vida, el $74 \%$ fue mala; la capacidad de autocuidado reporta que el $82 \%$ manifiesta autocuidados suficientes. Existe una correlación positiva
\end{abstract}

\footnotetext{
${ }^{1}$ Enfermero. Maestro en Enfermería. Profesor de Tiempo Completo Asociado "C" de la Facultad de Enfermería. Benemérita Universidad Autónoma de Puebla. Puebla, México. E-mail: alexanderreyes20102029@gmail.com ORCID ID: https://orcid.org/0000-0001-5133-4172 Autor principal - Endereço para correspondência: 4 Sur 104 Centro Histórico C.P. 72000 , Puebla, México.

2Enfermera. Maestra en Ciencias de Enfermería. Profesor de Tiempo Completo Asociado "C" de la Facultad de Enfermería. Benemérita Universidad Autónoma de Puebla. Puebla, México. E-mail: inesteq@gmail.com ORCID ID: https://orcid.org/00000002-3987-8254

${ }^{3}$ Enfermera. Maestra en Ciencias de Enfermería. Profesor de Tiempo Completo Asociado "C" de la Facultad de Enfermería. Benemérita Universidad Autónoma de Puebla. Puebla, México. E-mail: arachely60@hotmail.com ORCID ID: https://orcid.org/0000-0001-5343-4257

${ }^{4}$ Enfermera. Maestra en Ciencias de Enfermería. Profesor de Tiempo Completo Asociado "C" de la Facultad de Enfermería. Benemérita Universidad Autónoma de Puebla. Puebla, México. E-mail: erika.perez@correo.buap.mx ORCID ID: https://orcid.org/0000-0001-7058-0977

${ }^{5}$ Enfermero. Maestro en Enfermería. Profesor de Tiempo Completo Asistente Seniur de la Escuela de Enfermería. De la Universidad de las Américas Puebla. Puebla, México. E-mail: clemente.cordero@udlap.mx ORCID ID: https://orcid.org/0000$\underline{0002-3175-9664}$

${ }^{6}$ Estudiante de la Licenciatura en Enfermería. Benemérita Universidad Autónoma de Puebla. Puebla, México. E-mail: natalyrd19@gmail.com ORCID ID: https://orcid.org/0000-0003-2204-4269

${ }^{7}$ Enfermero. Maestro en Ciencias de Enfermería. Profesor de Tiempo Completo Asociado "C" de la Facultad de Enfermería. Benemérita Universidad Autónoma de Puebla. Puebla, México. E-mail: holistic-care21@hotmail.com ORCID ID: https://orcid.org/0000-0002-6772-4690
} meio, desde que a publicação original seja corretamente citada. 
y moderada entre calidad de vida y capacidad de autocuidado. Conclusión: el cáncer afecta más a mujeres predominando el de mama. Se encontró relación entre calidad de vida y capacidad de autocuidado, es decir, que a mayores capacidades de autocuidado mejor calidad de vida en los pacientes con cáncer.

Descriptores: Neoplasias; Autocuidado; Actividades Cotidianas; Calidad de Vida.

\section{ABSTRACT}

Objective: to determine the relationship of quality of life and self-care capacities in adult patients with cancer. Method: correlational, analytical, non-probabilistic convenience study with a sample constituted by 51 adult patients diagnosed with cancer who agreed to participate in the study through informed consent. A sociodemographic data card and two instruments were applied: Quality of Life WHOQOL-BREF and Capacity of Self-care Agency. Results: the average age was 50.32 years, the female gender predominated (60\%) and the types of cancer with the highest prevalence were: breast cancer (20\%), prostate cancer (16\%) and non-hodking lymphoma (12\%), other types (52\%). 80\% were in stage III TNM and in relation to quality of life, $74 \%$ was bad; self-care capacity reports that $82 \%$ show sufficient selfcare. There is a positive and moderate correlation between quality of life and ability to self-care. Conclusion: cancer affects more women and the predominant type is the breast cancer. A relationship was found between quality of life and ability to selfcare, that is, the greater self-care capacities are the better quality of life in cancer patients is.

Descriptors: Neoplasms; Self-Care; Daily Activities; Quality of Life.

\section{RESUMO}

Objetivo: determinar a relação entre qualidade de vida e capacidade de autocuidado em pacientes adultos com câncer. Método: estudo correlacional, analítico, não probabilístico por conveniência com uma amostra constituída por 51 pacientes adultos com diagnóstico de câncer, que aceitaram participar do estudo por meio de consentimento informado. Aplicou-se uma cédula de dados sociodemográficos e dois instrumentos: Qualidade de Vida WHOQOL-BREF e Capacidade de Autocuidado. Resultados: a idade média foi de 50,32 anos, do sexo feminino predominantemente $(60 \%)$, os tipos de cáncer com maior prevalencia foram: mama (20\%), de próstata (16\%) e linfoma não-Hodgkin (12\%), outros tipos (52\%). 80\% estavam em estágio III TNM, com relação à qualidade de vida, $74 \%$ era ruim; a capacidade de autocuidado reporta que $82 \%$ manifestam autocuidados suficiente. Existe correlação positiva e moderada entre qualidade de vida e capacidade de autocuidado. Conclusão: o câncer afeta mais mulheres, predominando o câncer de mama. Encontrou-se relação entre qualidade de vida e capacidade de autocuidado, ou seja, maior capacidade de autocuidado reflete em melhor qualidade de vida em pacientes oncológicos.

Descritores: Neoplasias; Autocuidado; Atividades Cotidianas; Qualidade de Vida.

\section{INTRODUCCIÓN}

La Organización Mundial de la Salud. Establece que el cáncer es la segunda causa de mortalidad a nivel mundial ${ }^{1}$. Atribuyendo 8,8 millones de defunciones, de las cuales las más frecuentes son: cáncer pulmonar representando 1,69 millones, 788000 por cáncer hepático, por cáncer gástrico 
754 000, por cáncer colorrectal 774, por cáncer mamario 571000 y 400000 por cáncer de esófago. Las patologías oncogénicas desarrolladas por virus tales como la Hepatitis B y C o Virus del Papiloma Humano, representan el 25\% de muertes por cáncer².

Existen ciertos factores de riesgo entre ellos: $30 \%$ asociado con el alto índice de masa corporal, pobre ingesta de verduras y frutas, escasa actividad física, consumo de alcohol y tabaco, este último es el principal factor de riesgo, en general representa más del $22 \%$ de defunciones ${ }^{3}$. El desarrollo de esta enfermedad también representa una afectación en la calidad de vida de la persona debido a que se limitan a ciertas funciones cuando la enfermedad se convierte en una situación crítica para el paciente. Se estima que en las próximas dos décadas se incremente el número de nuevos casos aproximadamente en un $70 \%$, generando de 14 a 22 millones de muertes $^{1,2}$.

Asimismo en la región de las Américas se registraron 2.8 millones de casos nuevos y 1,3 millones de muertes a consecuencia del cáncer, en el 2012. Las proyecciones indican que el número de muertes por cáncer en las Américas aumentará de 1,3 millones en el 2012 hasta 2,1 millones en el 2030. Las tasas de mortalidad por cáncer cervicouterino son unas 3 veces más altas en América Latina y el Caribe que en Norteamérica, poniendo de manifiesto la existencia de grandes desigualdades en salud. Mediante cambios en los estilos de vida y la adopción de hábitos más saludables sería posible evitar hasta un 30\% de las muertes por cáncer. La gran mayoría de las muertes por cáncer son prevenibles: cada año más de 260.200 personas mueren en las Américas a consecuencia del cáncer de pulmón, seguidos de, próstata y colorrectal en hombres, y mama y colón rectal en mujeres ${ }^{4}$.

Debido a este gran impacto que representa el cáncer, la Organización Mundial de la Salud y el Centro Internacional de Investigaciones sobre el Cáncer en colaboración conjunta con organizaciones que forman parte del equipo de tarea interinstitucionales de las Naciones Unidas sobre la Prevención y Control de las Enfermedades No Transmisibles crearon estrategias con la finalidad de prevención y control del cáncer, fortalecer los sistemas de salud para que presten servicios asistenciales y curativos a pacientes con cáncer, prolongar y mejorar la calidad de vida definida por la OMS como la percepción 
que un individuo tiene de su lugar en la existencia, relacionada con su cultura, valores, objetivos, expectativas, normas $e$ inquietudes en los que vive, influido por la salud física del mismo sujeto, su estado psicológico, nivel de independencia, relaciones sociales, así como su relación con los elementos esenciales de su entorno de vida del paciente de forma considerable $e^{3,4}$.

Por otra parte el Instituto Nacional del Cáncer (NCl), en su informe anual a la nación de Estados Unidos respecto a las cifras de cáncer determino que los índices de mortalidad e índices de incidencias por esta causa han ido en descenso en el periodo comprendido de 2003 a 2012 por cada 100000 habitantes lo que corresponde un promedio de 1,8\% al año en hombres y 1,4\% al año en mujeres. Situación que no sucede con el cáncer de hígado debido a que ha ido en aumento de 28,012 diagnósticos, de los cuales 20,207 fueron hombres y 7,805 mujeres. Por lo que continúan los estudios de investigación básica para prevenir y tratar el cáncer para prologar y mejorar la calidad de vida de las personas ${ }^{5}$.

En México, el Sistema Nacional de Vigilancia Epidemiológica reporta 78,719 muertes por cáncer, lo que representa una tasa general de 67.8 muertes por cada 100000 habitantes, las mujeres fueron el grupo más afectado con una tasa de 68.0 mientras que los hombres, presentaron una tasa de 67,5; donde los tipos de cáncer pulmonar, prostático, gástrico, hepático y mamario son los de mayor causa de mortalidad; así mismo refiere que se debe atender el estado psicológico de la persona que padece cáncer de tal forma que se maximice su calidad de vida y se fomente el autocuidado en conjunto con la de sus familiares y cuidadores ${ }^{6}$.

En el 2013, en el estado de Puebla, el Instituto Nacional de Estadísticas y Geografía e Informática (INEGI) reporta que once de cada 100 muertes registradas fueron causadas por algún tumor de los cuales el 92\% de ellos fueron causados por tumor maligno, por lo que representa un problema de salud debido a que las neoplasias son la cuarta causa de muerte en la población, esto representa en el sexo masculino la quinta causa de mortalidad; es decir $45.7 \%$ y para el sexo femenino la tercera causa de mortalidad, lo que representa 54.3\%, siendo el tabaco el factor de riesgo más importante, deteriorando así el 
funcionamiento adecuado del organismo ${ }^{7}$.

Por lo anterior, se sabe que el cáncer es un problema global de salud pública, donde se ecuentra comprometido el organismo del paciente, disminuyendo así las capacidades de autocuidado. En este sentido, Dorotea Orem, define al autocuidado; como aquella actividad que los individuos aprenden y que son ejecutadas en situaciones concretas de la vida y van dirigidas hacia un mismo fin que permite a la persona ser independiente y promover las condiciones necesarias para preservar la vida en beneficio de su salud $y$ bienestar. Donde también se debe de contar con tres elementos: Capacidades fundamentales y disposición del autocuidado: considerados las habilidades básicas del individuo (percepción, memoria y orientación). Componente de poder: que está constituido por habilidades que impulsan el individuo a la acción de auto cuidarse (motivación, adquisición de conocimientos, habilidad para ordenar acciones de autocuidado y capacidad de integración del autocuidado en su vida familiar y comunitaria). Capacidad de operacionalizar el autocuidado: que corresponde a las habilidades que le permiten al individuo investigar sobre condiciones de sí mismo y del medio ambiente, que son significativos para su autocuidado, así como la toma de decisiones y construcción de las acciones para lograr el manejo de su enfermedad ${ }^{8}$.

De acuerdo a la pirámide de Maslow según la OMS, las acciones del ser humano están motivadas para cubrir ciertas necesidades, requieren apoyo, seguridad, sentirse queridos, ser independientes y necesitan afecto. Conforme se satisfacen las necesidades básicas, los seres humanos desarrollan necesidades y deseos más elevados. Cuando un paciente es diagnosticado por esta patología, ya sea en fase temprana o terminal coexisten múltiples reacciones emocionales 0 síntomas intensos debido a diversos factores que, además, causan en él un cierto grado de incapacitación, preocupación e impacto emocional viéndose así alteradas todas sus dimensiones biológicas, psicológica, familiar, social y laboral, reflejándolo en la disminución de su autocuidado y afectando su calidad de vida ${ }^{1-2}$.

Con lo anterior algunos unos autores han abordado la calidad de vida en personas sobrevivientes de 
Carcinoma in situ (DCIS). Encontrando que las mujeres que mantuvieron un IMC saludable tuvieron en promedio un puntaje mayor que las mujeres con obesidad (normal versus obesidad: $B=$ 3.02; 2.18, 3.85). Los puntajes físicos de la calidad de vida también se elevaron entre aquellos que eran físicamente activos $(5+h$ / semana vs. ninguno: $B=1.96 ; 0.72,3.20)$, aquellos que consumían al menos siete bebidas / semana de alcohol (vs. ninguno; $B=$ $1.40 ; 0.39,2.41$ ), y no fumadores (vs. fumadores actuales: $B=1.80 ; 0.89$, 2.71). La calidad de vida mental fueron significativamente más altas entre las mujeres que eran moderadamente activas físicamente (hasta $2 \mathrm{~h} / \mathrm{semana}$ vs. ninguna: $B=1.11 ; 0.30,1.92$ ) y las no fumadoras (vs. los fumadores actuales: $B=1.49 ; 0.45,2.53)^{9}$.

Asimismo otros investigadores han evidenciado que la calidad de vida en relación a las dimensión del funcionamiento físico; $41 \%$ de las mujeres refirió tener dificultad para dar un paseo largo; $18 \%$ tiene dificultad para realizar esfuerzos importantes como llevar una bolsa de compra o una maleta, $15 \%$ tuvo impedimento importante para hacer su trabajo. A su vez $13,2 \%$ tuvo limitaciones para sus actividades de ocio y entretenimiento $n$ relación al estado emocional, 73\% de las mujeres refirió sentirse preocupada, 64\% sentirse nerviosa, deprimida $46 \%$ y 43\% irritable. $27 \%$ de las mujeres estaba bastante o muy preocupada en relación a todo lo que concierne a la enfermedad y $23 \%$ bastante o muy nerviosa. En el desenvolvimiento social, $79,2 \%$ de las encuestadas indicó que no interfirió su estado físico o su tratamiento médico en su vida familiar, por el contrario todas las encuestadas comentaron el apoyo permanente de familiares y amigos. En las escalas de salud general y calidad de vida las mujeres, el $40 \%$ valora su salud general como buena o muy buena (18\%) y $48 \%$ valora su calidad de vida como buena o muy buena (18\%) (RECALDE) ${ }^{10}$.

Otros autores abordaron las capacidades de autocuidado en pacientes con enfermedad crónica hallaron que los participantes posee alta capacidad de autocuidado. En lo que corresponde a la categoría de interacción social, se demostró que 11 de los participantes poseen alta capacidad de agencia de autocuidado, mientras que 2 de las personas están dentro de baja capacidad de autocuidado. En relación con la categoría de consumo suficiente de alimentos, cinco personas poseen baja 
capacidad de agencia de autocuidado. Finalmente, en las categorías de bienestar personal, actividad y reposo, así como funcionamiento y desarrollo personal, el total de la población se ubicó en el parámetro alta capacidad de autocuidado ${ }^{11}$.

La atención de pacientes oncológicos es llevada a cabo por profesionales de la salud, por lo cual, la disciplina de enfermería tiene la necesidad y convicción de brindar cuidados integrales; debido a que se ubica en la primera línea de atención a la salud, en este sentido el objetivo del estudio es determinar la relación de calidad de vida y autocuidado en pacientes adultos con cáncer.

\section{MÉTODO}

El diseño del estudio es de tipo descriptivo, de corte transversal, correlacional y analítico. El muestreo es no probabilístico por conveniencia y el cálculo de la muestra se obtuvo mediante la fórmula de varianza con un poder estadístico de 0.80 y un coeficiente de correlación de 0.20, teniendo una muestra $(n=51)$. Para amortiguar el efecto de varianza, se incrementó un 10\%, quedando conformada por una 80 personas con diagnóstico de cáncer ${ }^{12}$.

La muestra estuvo conformada

por 51 pacientes adultos con diagnóstico de cáncer de un hospital de tercer nivel de atención de la ciudad de Puebla. Los criterios de inclusión fueron: adultos de 18 a 59 años de edad, que se encuentren en los servicios de oncología hospitalización, oncología quirúrgica, oncología ambulatoria y oncología de consulta externa de un hospital de tercer nivel de atención de la ciudad de Puebla y que bajo consentimiento informado aceptaron participar en el estudio. Criterios de exclusión; pacientes pediátricos con cáncer, pacientes post-trasplantados con rechazo a medula espinal.

El Primer instrumento utilizado fue el Cuestionario Calidad de Vida de WHOQOL-BREF, elaborado por un grupo de investigadores de la Organización Mundial de la Salud con la finalidad de obtener una definición consensuada de calidad de vida y aplicarla en todas las cultura $^{13}$. El instrumento consta de 26 Ítems; dos preguntas generales sobre calidad de vida y satisfacción con el estado de salud, y 24 preguntas, cada ítem tiene 5 opciones de respuesta tipo Likert (1 corresponde a nada, 2 un poco, 3 lo normal, 4 bastante y 5 
extremadamente).están agrupadas en cuatro áreas: Salud Física, Salud Psicológica, Relaciones Sociales y Ambiente. Ha sido traducido a 19 idiomas diferentes, es aplicable a sujetos sanos y enfermos, y su puntaje ha sido utilizado para observar los cambios en la calidad de vida debido a la enfermedad, para establecer valores pronósticos de la enfermedad y para valorar algunas intervenciones. Este instrumento fue validado em el español de México, por Centro Universitario de Ciencias donde se obtuvo un coeficiente de alfa de Cronbach de 0.88.

El segundo instrumento fue el de la Capacidad de Agencia de Autocuidado en el Paciente Adulto con Cáncer está basado en la Teoría de Autocuidado de Dorotea Orem. Se agrupa en tres dimensiones: $1 . \quad$ Capacidades fundamentales y disposición del autocuidado, 2. Componentes de poder y 3. Capacidad de operacionalizar el autocuidado. Fue elaborado por Universidad Javeriana Bogotá Colombia, validado al contexto de México por Gallegos E; con un alpha de cronbach de $0.80^{14}$. Evalúa las habilidades que impulsan al individuo a la acción de autocuidarse. Consta de 17 items agrupados en tres dimensiones: I. Capacidades fundamentales (comprende los ítems 1,2 y 3); II. Componentes de poder (4, 5, 6 y 7); y III. Capacidad para operacionalizar $(8,9,10,11,12,13$, $14,15,16$ y 17). Cuenta con una escala de respuesta tipo Likert con cinco opciones de respuesta, donde $1=$ nunca, $2=$ casi nunca, $3=a$ veces, 4= casi siempre y $5=$ siempre.

La recolección de la información se realizó en un Institución de Salud de tercer Nivel de Atención, una vez que se obtuvo la aceptación del participante elegido se procedió a solicitar la firma de consentimiento informado para posteriormente pedirle el llenado de los dos cuestionarios. Esta investigación se llevó a cabo de acuerdo a las disposiciones de la Ley General de Salud en materia de investigación para la salud de México; así mismo, se obtuvo la aprobación por el Comité de revisor de Investigación de la Universidad Popular Autónoma del Estado de Puebla (UPAEP) con el número de folio Durante la aplicación del instrumento, se tomaron las medidas necesarias que permitieron respetar la privacidad de los pacientes, bajo los principios básicos de ética: autonomía, beneficencia, no maleficencia y justicia ${ }^{15}$.

\section{RESULTADOS}


El $70 \%$ de la muestra se encuentra en un rango de edad de 40 a 82 años; la relación de género de masculino a femenino es de 1:2, respecto a la ocupación más del $30 \%$ de los pacientes son profesionistas, predomino la escolaridad, de licenciatura; así mismo se puede observar que los tipos de cáncer de mayor influencia fueron: el cáncer de mama (20\%), próstata (16\%), linfoma no Hodking (12\%), cáncer cervicouterino (10\%) y leucemia linfoblástica aguda (10\%), representando un $68 \%$ de los pacientes participantes, cabe mencionar El $80 \%$ estuvo en estadio III del sistema de estadificación de cáncer TNM, donde, más de la mitad del género femenino fue afectada, de este grupo más del 30\% refiere tener 2 años padeciendo la enfermedad.

El tratamiento médico más utilizado por los pacientes es la quimioterapia con un $80 \%$, solo el $2 \%$ es tratado con radioterapia y el $14 \%$ ambos tratamientos. La familia representa el soporte vital para estos pacientes debido a que más del $60 \%$ de ellos vive en familia y el cuidado es proporcionado por el núcleo familiar. Más del $90 \%$ de la muestra refirió no contar con una red de apoyo de pacientes con cáncer, así como también más del $80 \%$ son católicos.

Se observa en la tabla1 la confiabilidad de los instrumentos utilizados.

Tabla 1 - Coeficiente de Confiabilidad. ( $n=51)$

\begin{tabular}{lcc}
\hline$N^{\circ}$ & $\begin{array}{c}N^{\circ} \text { de } \\
\text { Reactivos Cronbach }\end{array}$ & $\begin{array}{c}\text { Alpha de } \\
\text { Reactivos }\end{array}$ \\
1 Escala de Calidad de Vida & 26 & .93 \\
WHOQOL-BREF & & \\
$2 \begin{array}{l}\text { Instrumento para evaluar } \\
\text { la Capacidad de Agencia } \\
\text { de Autocuidado en }\end{array}$ & 17 & .76 \\
Pacientes Adultos con & & \\
\end{tabular}

La consistencia interna del instrumento se determinó con el coeficiente de confiabilidad Alpha de Cronbach, que es aceptable de acuerdo a Polit \& Beck ${ }^{12}$.

Tabla 2 - Calidad de Vida de los pacientes Adultos con Cáncer. $(n=51)$

\begin{tabular}{clcc}
\hline$N^{\circ}$ & \multicolumn{1}{c}{ Variable } & $f$ & $\%$ \\
1 & Mala calidad de vida & 37 & 74 \\
2 & $\begin{array}{l}\text { Buena calidad de } \\
\text { vida }\end{array}$ & 13 & 26 \\
\hline
\end{tabular}

Tabla 3 - Capacidad de Autocuidado en pacientes Adultos con Cáncer. $(n=51)$

\begin{tabular}{|c|c|c|c|}
\hline$N^{\circ}$ & Variable & $f$ & $\%$ \\
\hline 1 & $\begin{array}{l}\text { Autocuidados } \\
\text { deficientes }\end{array}$ & 9 & 18 \\
\hline 2 & $\begin{array}{l}\text { Autocuidados } \\
\text { suficientes }\end{array}$ & 41 & 82 \\
\hline
\end{tabular}

Se puede observar que, la calidad de vida de los pacientes adultos con cáncer; es mala versus buena en una razón de 2:1, esto indica que más del $70 \%$ de la muestra presenta mala calidad de vida. 
Los autocuidados que tienen los pacientes adultos con cáncer; es suficiente versus deficiente en una razón de 4:1, esto indica que más del $80 \%$ de la muestra presenta un autocuidado suficiente de su enfermedad.

Para cumplir con el objetivo del estudio y al obtener distribución normal de las variables continuas, calidad de vida y capacidades de autocuidado, por medio de la prueba de Kolmogorov Smirnov $(p s \quad=\quad .85$ y .14 , respectivamente), se realizó la prueba de Spearman entre ambos índices son positivos y moderados ${ }^{12}$.

Tabla 4 - Matriz de correlación de calidad de vida y capacidades de autocuidado. $(n=51)$

\begin{tabular}{cccc}
\hline $\mathbf{N}$ & Variable & 1 & $\mathbf{2}$ \\
0 & & 1 & \\
1 & Indice de Calidad de Vida & 1 & \\
2 & $\begin{array}{l}\text { Indice de Capacidades de } \\
\text { Autocuidado }\end{array}$ & $.632^{* *}$ & 1 \\
\hline
\end{tabular}

Se encontró que en los casos de autocuidado suficientes, hubo mejor la calidad de vida en las pacientes con cáncer $\left(r_{s}=0,632 p<0,005\right)$ Lo anterior muestra una correlación positiva de acuerdo a la tendencia del diagrama de la figura 1, lo que se traduce en que las capacidades fundamentales y disposición del autocuidado: considerados las habilidades básicas impulsan al paciente a la acción de auto cuidarse con ello mejorar su calidad de vida.

Figura 1. Diagrama de Regresión Lineal Simple.

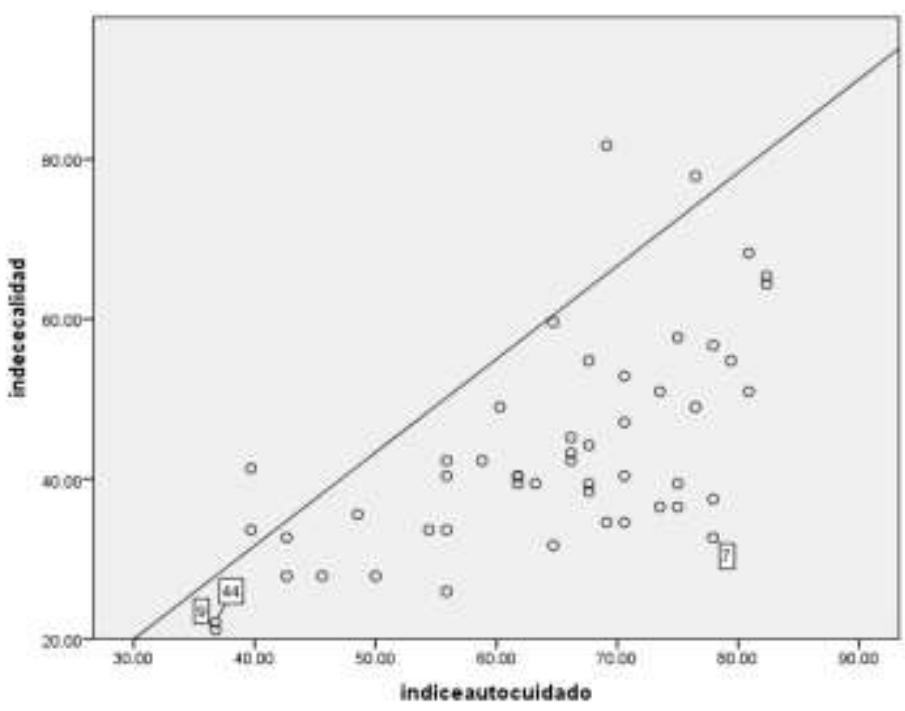

regresión lineal simple, se aprecia algunos valores extremos, como es el número 7, 9 y 44. Donde el paciente numero 7 presenta características diferentes al de los demás datos como género femenino, madre soltera, escolaridad licenciatura, vivir sola, lo cuida su familia y padecer cáncer desde hace 1 año 3 meses, el adulto 9 es género femenino, ser soltera, escolaridad secundaria, vivir sola, le cuidan sus hijos y padecer cáncer hace 3 meses, el adultos 44 es femenino, soltera, escolaridad preparatoria, vive con la familia, le cuidan sus hijos y padece cáncer desde hace 6 meses. Características que no se presentan de forma agrupada en los demás adultos, lo que significa que monitorear a personas con estas características, 
permite al profesional de enfermería fortalecer su capacidad de autocuidado.

\section{DISCUSIÓN}

El presente estudio tuvo como objetivo en determinar la relación de calidad de vida y capacidades de autocuidado en pacientes adultos con cáncer. Como marca la literatura el cáncer es un problema de salud pública tanto a nivel mundial, internacional y nacional ${ }^{2,3,4}$; generando cambios en el autocuidado de las personas que viven este proceso de enfermedad, viéndose afectadas las dimensiones físicas, psicológicas, social y espiritual.

Por otro lado, el impacto que representa el cáncer de morbilidad y mortalidad a nivel mundial la OMS y el Centro Internacional de Investigaciones sobre el Cáncer y las Naciones Unidad sobre la Prevención y Control de las Enfermedades No Transmisibles han creado estrategias sobre el control y prevención del cáncer, se han fortalecido los sistemas de salud para la prestación de servicios asistenciales y curativos a pacientes con cáncer, para prolongar y mejorar la calidad de vida ${ }^{3,4}$; sin embargo, se encontró que prevalece más de la mitad de la muestra del estudio ha padecido cáncer, esto es concordante con datos de incidencia y mortalidad de mujeres que padecen cáncer en México esto reportado por el Sistema Nacional de Vigilancia Epidemiológica (SINAVE) ${ }^{6}$.

Es por ello, como acota Orem ${ }^{8}$ en su modelo refiere que la persona cuenta con tres elementos fundamentales que son: a) las capacidades de autocuidado y disposición del autocuidado, b) componente de poder y c) capacidad de integración del autocuidado. Con respecto a las capacidades de autocuidado, autores ${ }^{8}$ refieren que las prácticas de autocuidado mediante la intervención educativa de enfermería son elemento fundamental en la adaptación del paciente a mejorar su condición de salud y su calidad de vida, condición vital que requiere en este estudio para mejorar la calidad de vida de los pacientes.

En otro punto Recalde $y$ Samudio $^{9}$ encontró que las personas que reciben quimioterapia y radioterapia adoptan estrategias de autocuidado para hacer frente a la situación en que se encuentran y los efectos secundarios causados por los tratamientos. El reposo, técnicas de relajación y escuchar música son las estrategias para luchar con la fatiga, el estrés y la 
ansiedad. Una buena red de apoyo familiar, la buena gestión de la enfermedad y todas las consultas marcadas eran aspectos indicados como promotores de autocuidado a nivel físico y psicológico. El uso de medicinas alternativas para combatir los efectos secundarios fue también un factor encontrado como promotor de autocuidado.

El autocuidado es una función humana reguladora que los individuos tienen deliberadamente que desempeñar por sí mismos o que alguien lo ejecute por ellos para preservar la vida, salud, desarrollo y bienestar; por otro lado, enfermería educa sobre el autocuidado a pacientes en especial énfasis en tratamiento oncológico para que aprendan a manejarse por sí mismos y puedan sobrellevar la enfermedad y los efectos adversos que causa la quimioterapia, factor que concuerda a que en su mayoría de los pacientes se encontraron bajo tratamiento con quimioterapia ${ }^{8,9,10}$.

Por último, enfermería tiene las herramientas para brindar una atención de calidad, en cualquier situación relacionada con el binomio saludenfermedad tanto por personas enfermas, como por personas que están tomando decisiones sobre su salud, y por personas sanas que desean mantenerse o modificar conductas de riesgo para su salud, este modelo de Orem proporciona un marco conceptual y establece una serie de conceptos, definiciones, objetivos, para intuir lo relacionado con los cuidados de la persona ${ }^{8}$.

\section{CONCLUSIÓN}

El cáncer es una enfermedad grave, pero cuando se brinda el cuidado óptimo este se puede revertir, en este sentido, la calidad de vida de las personas con cáncer incluye múltiples dimensiones: físicas, sociales, psicológicas y espirituales, que el profesional de enfermería tendrá en cuenta en su plan de cuidados; las medidas de bienestar serán actividades funcionales de manejo del dolor, la fatiga, el cansancio, sueño, náuseas, vómitos y otros síntomas como respuestas individuales del paciente. Por lo que respecta a las capacidades de autocuidado se relacionan moderadamente con la calidad de vida en pacientes adultos con cáncer.

Por tanto, es considerando el autocuidado y la calidad de vida como piezas fundamentales para la promoción y la recuperación de la salud de los individuos, se apunta a la 
importancia de un trabajo encaminado para un cuidado integral en la salud con una mirada ampliada para los aspectos psicosociales en la salud. Algunos de los Hallazgos que se encontraron fue que las personas que mejoraron su capacidad de autocuidado perciben mejor su calidad de vida.

Algunas de las limitaciones durante el trabajo de investigación fueron los efectos secundarios de la enfermedad, que se presentaron durante la aplicación de los instrumentos, lo que limito la muestra, así como los accesos a las instituciones gubernamentales para la realización del trabajo debido a que aún falta cultura por la investigación. Algunas perspectivas para estudios futuros es realizar estudios longitudinales, así como de efecto en las intervenciones de enfermería para incrementar la capacidad de autocuidado.

\section{REFERENCIAS}

1. Organización Mundial de la Salud. Cáncer. Washington: OMS; 2018 [acceso en 2018 jul 12]. Disponible en: http://www.who.int/es/newsroom/fact-sheets/detail/cancer

2. Organización Mundial de la Salud. Global action plan for the prevention and control of noncommunicable diseases 2013-2020. Washington: OMS; 2018 [acceso en 2018 sept. 14]. Disponible en: https://bit.ly/2PMB1lR 3. World Health Organization. World Cancer Report. Ginebra: OMS; 2014 [acceso en 2018 ene. 10]. Available en: http://publications.iarc.fr/NonSeries-Publications/World-CancerReports/World-Cancer-Report-2014

4. Organización Panamericana de la Salud. Cáncer en las américas perfiles de país. Washington: OPS; 2013. [acceso en 2018 feb. 11]. Disponible en:

https://www.paho.org/hq/dmdocum ents/2014/Perfiles-Pais-Cancer-2013ESP.pdf

5. Instituto Nacional de Cáncer. Sumarios del PDQ de información sobre el cáncer. México: INC; 2016. [acceso en 2018 mar. 14]. Disponible en:

https://www.cancer.gov/espanol/pu blicaciones/pdq/sumarios-deinformacion

6. Sistema Nacional de Vigilancia Epidemiológica. Boletín Epidemiológico. México: SISVEA; 2018. [acceso en 2018 nov. 22]. Disponible en: https://www.gob.mx/cms/uploads/a ttachment/file/413436/sem45.pdf 
7. Instituto Nacional de Estadística y Geografía. Estadísticas a propósito del día mundial contra el cáncer. México: INEGI; 2018. [acceso en 2018 nov. 8]. Disponible en: http: //www.beta.inegi.org.mx/conte nidos/saladeprensa/aproposito/2018/ cancer2018_Nal.pdf

8. Prado-Solar LA, González-Reguera M, Paz-Gómez N, Romero-Borges K. La teoría déficit de autocuidado: Dorothea Orem punto de partida para calidad en la atención. Rev Med Electron. 2014; 36(6):835-45.

9. Recalde MTI, Samudio M. Calidad de vida en pacientes con cáncer de mama en tratamiento oncológico ambulatorio en el Instituto de Previsión Social en el año 2010. Mem Inst Investig Ciênc Salud. 2014; 10(2):13-29.

10. Leiva $V$, Acosta $P$, Berrocal $Y$, Carrillo E, Castro M, Watson Y. Capacidad de agencia de autocuidado en las personas adultas que padecen artritis reumatoide. Enferm Actual Costa Rica. 2012; 22:1-11.

11. Achury DM, Sepúlveda GJ, Rodríguez SM. Instrumento para evaluar la capacidad de agencia de autocuidado en pacientes con hipertensión arterial. Investig enferm. 2009; 11(2):9-25.

12. Polit DF, Beck CHT. Canadian Essentials of Nursing Research. Wolters Kluwer: Fourth edition; 2018.

13. López-Huerta JA, González-Romo RA, Tejada-Tayabas JM. Propiedades Psicométricas de la Versión en Español de la Escala de Calidad de Vida WHO QoL BREF en una Muestra de Adultos Mexicanos Rev iberoam diagn eval psicol. 2017; 44(2):105-15.

14. Gallegos Cabriales E. Validez y confiabilidad de la versión en español de la escala: valoración de las capacidades de auto-cuidado Desarro. Cient enferm. 1998; 6(9):260-6.

15. Secretaría de Salud. Reglamento de la ley general de salud en material de investigación para la salud. México: SSA; 1987. 
Conflito de interesses: Os autores declaram não haver conflito de interesses.

\section{Participação dos autores:}

- Concepção: García-López MA, Pérez-Noriega E, Ramos-Durán N, MoralesCastillo FA.

- Desenvolvimento: Torres-Reyes A, Cordero-Sánchez C, Ramos-Durán N, Morales-Castillo FA.

- Redação e revisão: Torres-Reyes A, Tenahua-Quitl I, Morales-Castillo FA.

Como citar este artigo: Torres-Reyes A, Tenahua-Quitl I, GarcíaLópez MA, Pérez-Noriega E, Cordero-Sánchez C, Ramos-Durán N, et al. Relación de calidad de vida y autocuidado en pacientes adultos con cáncer. J Health NPEPS. 2019; 4(1):16-30.

Submissão: $21 / 09 / 2018$

Aceito: $15 / 01 / 2019$

Publicado: 01/06/2019 